This item was submitted to Loughborough's Research Repository by the author.

Items in Figshare are protected by copyright, with all rights reserved, unless otherwise indicated.

\title{
Normal modes of a small gamelan gong
}

PLEASE CITE THE PUBLISHED VERSION

http://dx.doi.org/10.1121/1.4895683

\section{PUBLISHER}

() Acoustical Society of America

VERSION

VoR (Version of Record)

LICENCE

CC BY-NC-ND 4.0

REPOSITORY RECORD

Perrin, R., Daniel P. Elford, Luke Chalmers, G.M. Swallowe, Thomas R. Moore, Sinin Hamdan, and Ben J. Halkon. 2019. "Normal Modes of a Small Gamelan Gong”. figshare. https://hdl.handle.net/2134/18790. 


\title{
Normal modes of a small gamelan gong
}

\author{
Robert Perrin \\ Institute for Fundamental Sciences, Massey University, Palmerston North, New Zealand \\ Daniel P. Elford, Luke Chalmers, and Gerry M. Swallowe ${ }^{\text {a) }}$ \\ Department of Physics, Loughborough University, Loughborough, LE11 3TU, United Kingdom
}

Thomas R. Moore

Department of Physics, Rollins College, Winter Park, Florida 32789

Sinin Hamdan

Faculty of Engineering, Universiti Malaysia Sarawak (UNIMAS), Sarawak, Malaysia

Benjamin J. Halkon

School of Mechanical and Manufacturing Engineering, Loughborough University, Loughborough LE11 3TU, United Kingdom

(Received 19 November 2013; revised 16 June 2014; accepted 21 August 2014)

\begin{abstract}
Studies have been made of the normal modes of a $20.7 \mathrm{~cm}$ diameter steel gamelan gong. A finiteelement model has been constructed and its predictions for normal modes compared with experimental results obtained using electronic speckle pattern interferometry. Agreement was reasonable in view of the lack of precision in the manufacture of the instrument. The results agree with expectations for an axially symmetric system subject to small symmetry breaking. The extent to which the results obey Chladni's law is discussed. Comparison with vibrational and acoustical spectra enabled the identification of the small number of modes responsible for the sound output when played normally. Evidence of non-linear behavior was found, mainly in the form of subharmonics of true modes. Experiments using scanning laser Doppler vibrometry gave satisfactory agreement with the other methods. (C) 2014 Acoustical Society of America.
\end{abstract}

[http://dx.doi.org/10.1121/1.4895683]

PACS number(s): 43.75.Kk, 43.40.At, 43.40.Le [KML]

Pages: $1942-1950$

\section{INTRODUCTION}

A gamelan is defined as an ensemble of traditional musical instruments from the general region of the Indonesian archipelago. While several different types of instruments are involved, the backbone of the gamelan consists of idiophones and gongs. The latter come in a wide range of sizes and are traditionally made of bronze, although steel is now sometimes used. The gamelan is central to the musical art of Indonesia $^{1}$ where it commands huge respect and even reverence.

Compared to Western percussion instruments, there has been little attention paid to gamelan gongs in the scientific literature; a useful summary has, however, been given by Rossing. ${ }^{2}$ There have been further studies on large gamelan gongs, ${ }^{3}$ which are of particular interest because they show marked non-linear behavior. Apart from a preliminary report on the present work, ${ }^{4}$ there is only one further study on small gamelan gongs of which the authors are aware. ${ }^{5}$ This used finite-element models and acoustic measurements (only) to investigate the influences of various aspects of gong geometry on tuning.

In the present paper the results of a study on a small steel gamelan gong from Sarawak are reported. These gongs are very similar in geometry to the Indonesian ones but,

\footnotetext{
a) Author to whom correspondence should be addressed. Electronic mail: G.M.Swallowe@lboro.ac.uk
}

rather than being cast in bronze, are hammered into the desired shape starting from flat uniform circular metallic plates. Finite-element modeling (FEM) (Elford and Chalmers), electronic speckle pattern interferometry (ESPI) (Moore, Elford, and Chalmers) and laser Doppler vibrometry (LDV) (Halkon, Hamden, and Swallowe) have been used together with group representation theory (Perrin) and some acoustical measurements (Halkon, Swallowe, and Perrin).

\section{GONG GEOMETRY}

Although gamelan gongs come in a wide range of sizes, they all have a similar general form. Figure 1 shows the experimentally measured half cross-section of the small gamelan gong used in the present study taken through its center when placed on a horizontal surface. The gong consists of a central dome A on top of a roughly flat circular plate which is terminated by a shoulder BC and then a deep inward-sloping rim $\mathrm{CD}$. It has a basic axial symmetry with axis AE. The diameter at the widest point was $20.7 \mathrm{~cm}$ and the thickness of the metal was nominally $1.5 \mathrm{~mm}$. From the figure it can clearly be seen that the thickness is slightly less in regions of high bending due to its having been hammered out from a flat plate. Figure 2 shows a photograph of the gong in which the irregular nature of the surface can clearly be seen.

Gamelan gongs are normally rung by being struck on the central dome with a soft-headed mallet. Large gongs are 


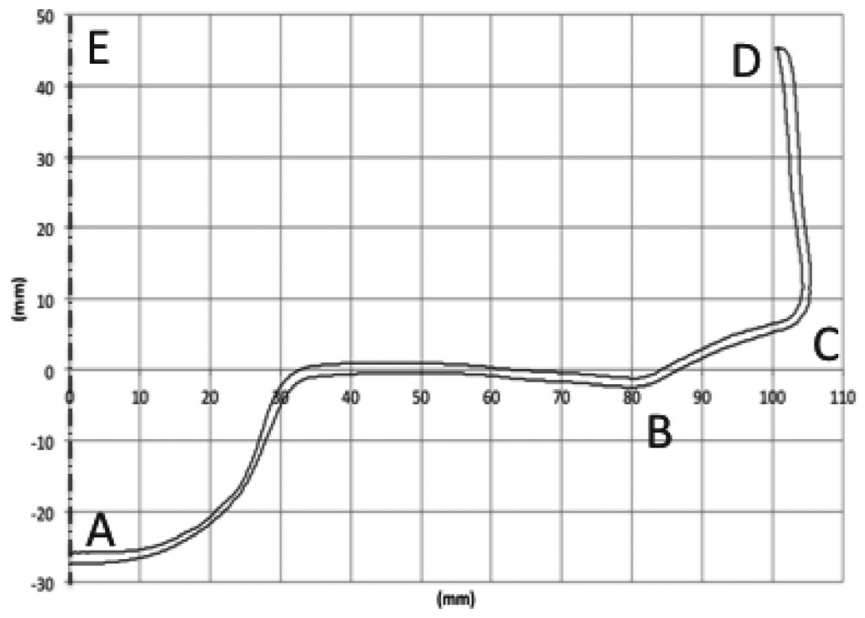

FIG. 1. Vertical cross-section through the small gamelan gong.

suspended vertically by strings; smaller ones are often orientated horizontally by sitting them on parallel pairs of horizontal strings.

\section{THEORETICAL CONSIDERATIONS}

Because of the gong's basic axial symmetry it is convenient to describe it using cylindrical polar coordinates with the $z$-direction lying along AE. Displacements in the $(r, \theta, z)$ directions will be referred to as $(u, v, w)$.

\section{A. The unperturbed gong}

As with other three-dimensional systems, the normal modes of gamelan gongs will have few, if any, true nodes (points of zero displacement). However, the individual $(r, \theta, z)$ components of the motion may do so. Since acoustical radiation is produced primarily by motion normal to the surface it has become the convention to describe the modes of bells in terms of the nodal patterns of their $r$-components. Likewise for cymbals it is usual to use the $z$-components. In the present case it is convenient to use the $z$-component on the upper parts of the gong and the $r$-component for the rim. The nodal patterns for a given mode for these regions can be expected to marry together smoothly due to overall symmetry requirements.

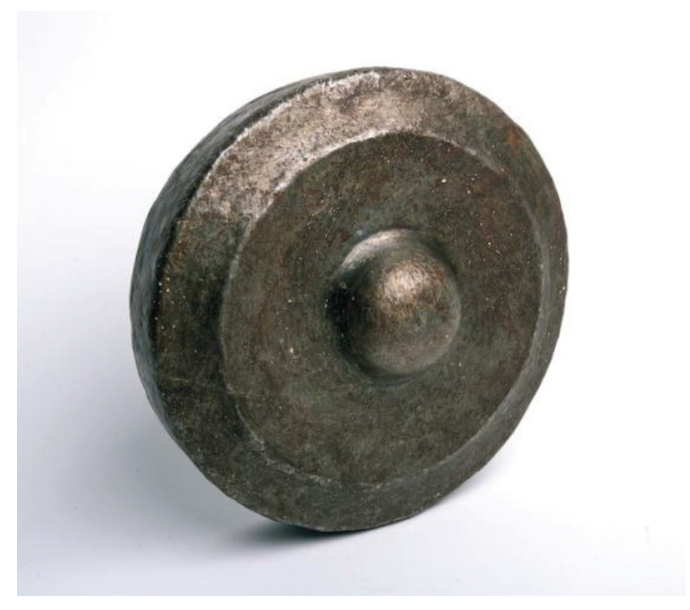

FIG. 2. (Color online) Photograph of the gong.
A truly axially symmetric gong is subject to the same consequences for its normal modes as are other systems with the symmetry group $C_{\infty v}$ such as bells, ${ }^{6}$ cymbals, ${ }^{7}$ and flat circular plates. ${ }^{8}$ Since the modal functions form a complete orthonormal set giving bases for all the irreducible representations of the group, these must vary like $\sin (m \theta)$ or $\cos (m \theta)$, where $m=0,1,2, \ldots$, giving degenerate pairs of modes. The case of $m=0$ is exceptional in having singlet axisymmetric modes. Thus the nodal patterns must consist of $m$ equally spaced "diameters" and $n$ circles parallel to the rim, the diameters of one doublet member lying mid-way between those of its partner. The number pair $(m, n)$ can be used to specify a degenerate pair of modes of a particular physical type. If it is desired to specify a particular member of a doublet this can be done by adding a subscript outside the bracket. It is possible to have modes of different physical types with the same $(m, n)$ values, equally well permitted by symmetry considerations but very different in frequency, as will be discussed in Sec. III B. All these arguments apply equally well to the $(r, \theta, z)$ components of the motion individually.

If the gong were truly axially symmetric then the actual locations of the nodal diameters would be indeterminate until fixed by initial conditions. However, since the gongs are cast or formed, there are always imperfections both of geometry and of metallurgy. These imperfections result in the doublets being split and the locations of their nodal diameters being fixed. Beats can subsequently be expected to occur as in the case of bell "warble." The gong used in the present study deviated considerably from perfect axial symmetry, resulting also in significant distortions in some of the nodal patterns.

\section{B. Extensional and inextensional modes}

The study of bells ${ }^{9}$ and other axially symmetric systems, ${ }^{10}$ as well as the ideas of Rayleigh, ${ }^{11}$ lead one to expect that the lowest frequency modes will always involve inextensional distortions of the system because this minimizes the potential energy of displacement. In the present case this means that if one takes a section through the gong at fixed $\mathrm{z}$ the resulting ring will contain a neutral circle whose total length remains unchanged throughout the cycle. This requires that the radial and transverse components of the motion to be related by

$$
u+\frac{\partial v}{\partial \theta}=0
$$

Thus, using the parts of the modal functions introduced in Sec. III A, one member of a degenerate pair may be written $u=m A \sin (m \theta)$ and $v=A \cos (m \theta)$, where $A$ is an arbitrary constant. So the modes have radial components that become increasingly larger than the transverse ones as $m$ increases. These inextensional modes will appear in an infinite series as one goes to higher frequencies. They will, however, eventually become supplemented by others, equally well-allowed by symmetry requirements, satisfying a complementary "extensibility" condition 


$$
v+\frac{\partial u}{\partial \theta}=0
$$

This results in modes whose transverse components are $m$ times their radial ones. Thus for every inextensional $(m, n)$ pair there is a corresponding extensional one which is an order of magnitude higher in frequency due to the increase in potential energy from the extension. These "extensional" modes are well-established as occurring in church bells ${ }^{12}$ and Indian elephant bells ${ }^{13}$ but seem unlikely to appear in small gongs at frequencies low enough to be of interest.

\section{FINITE-ELEMENT MODEL (FEM)}

\section{A. Construction of the model}

The structural mechanics module of COMSOL MULTIPHYSICS was used to investigate the normal modes of the gong. The inner and outer profiles for the half cross-section of the gong from the center of the dome $\mathrm{A}$ to the bottom of the rim $\mathrm{D}$ (see Fig. 1) were measured separately at a fixed value of $\theta$ using a metric coordinate measurement machine. This was repeated for a number of different orientations. An average was then taken for the outer half profiles and another for the inner ones. These were fed into COMSOL as geometric data and used to generate a three dimensional model with perfect axial symmetry. The facilities for automatic element selection and meshing were employed while the material properties were taken to be those of standard mild steel. A constraint was imposed on the top of the rim to mimic a clamp which proved necessary when making the ESPI measurements. This broke the axial symmetry slightly and so was expected to cause small splits in the model's predictions for the degenerate pairs.

\section{B. FEM results and discussion}

The eigenfrequency analysis facility of the COMSOL package was used to calculate the frequencies and display the modal forms of all modes it could find up to about $6 \mathrm{kHz}$. In most cases it was easy to identify a mode's value of $m$ by looking at the behavior of the top plate. However, this became more difficult as $m$ increased because a region of evanescence extended ever further away from the dome. A preliminary study suggested that the modes might be divided into "rim" and "plate" types. However, a comparison with results previously reported for cymbals ${ }^{7}$ made it clear that the "rim" modes are just $n=0$ cases for the complete gong where evanescence has "forced" the observable motion for all modes with $m \geq 3$ down onto the rim. "Plate" modes are those with $n \geq 1$ where the evanescent region is relatively small.

The symmetry type of a mode is determined by its value of $m$. The FEM correctly predicted all modes with $m=0$ to be singlets and all others to be doublets (slightly split as anticipated due to the point support). Some examples are given in Fig. 3 where a "rim" mode and an orthogonal pair of "plate" modes are shown in the top row. On the bottom row the three modes which proved to be the most important acoustically are shown.

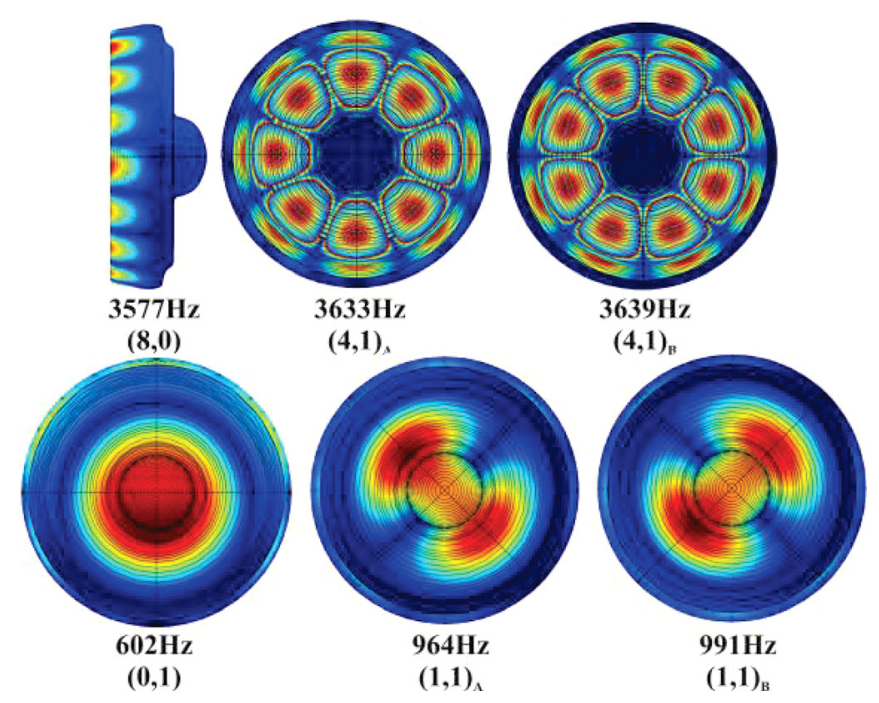

FIG. 3. (Color online) Selected modes predicted by the finite-element model.

\section{EXPERIMENTAL INVESTIGATION AND DISCUSSION}

\section{A. ESPI measurements}

In order to compare the FEM predictions with experiment the operational deflection shapes were studied using ESPI, ${ }^{15}$ a technique well-known for the visualization of vibrational modes of musical instruments. ${ }^{16,17}$ The facility employed has been described in detail elsewhere. ${ }^{18}$ The gong was mounted on a vibration-isolated optical table inside an anechoic chamber and excited by a loudspeaker placed about $50 \mathrm{~cm}$ behind it. The speaker was driven using a sinusoidal signal generated by a high-quality function generator /amplifier. The drive signal was carefully monitored in order to avoid introducing harmonic and subharmonic frequency content. Unfortunately the system did not permit the gong to be supported horizontally, as it would be during normal playing. Instead it was hung vertically, clamped to a stand along a small section near the center of the rim. This would certainly have influenced some of the modes and had to be taken into account when constructing the FEM and when interpreting the results. The model included fixed boundary conditions at the same point on the rim where the gong was clamped in the experiments in order to make the modeling and experimental conditions equivalent.

The identification of modes from their ESPI images was much more difficult than those from FEM. First the gong proved to have non-linear properties resulting in the appearance of both harmonics and subharmonics of many true modes as well as some mixed-symmetry types. There were also, as expected and especially for cases with $n \geq 2$, some serious pattern distortions. This was no doubt due to the influence of the irregularities from hammering which were largely smoothed out in the FEM because of the way in which its precisely axially symmetric form was generated. As the wavelengths involved in the patterns became smaller so the modes were more sensitive to the irregularities. Experiments looking at the top of the gong along the symmetry axis were not sufficient because, for modes with $n=0$, no nodal patterns were observed on the top plate for $m \geq 3$. 
However, looking from the side, modes with $(m, 0)$ for $m$ up to 10 were readily identified. Modes with $n=1$ were relatively easy to identify because their single nodal circle occurred at or near the inner edge of the shoulder. While a few modes with $n \geq 2$ were identified, this became increasingly problematic as $n$ increased. It was decided to pay them relatively little attention.

Figure 4 shows the ESPI images for the same modes displayed in Fig. 3. Clearly the modal forms are in good agreement. It should be noted that the doublet pairs are somewhat split in frequencies, as expected. Side-views for the $(4,1)$ modes are included to show their lack of skirt motion. Higher frequency modes with $n>0$ behaved similarly. The $(8,0)$ shows considerable skirt motion but very little on the face. It, like other $n=0$ modes with $m \geq 3$, could not be identified by ESPI measurements from the front.

As an example of subharmonics appearing in the ESPI results the parent modes for the orthogonal pair $(6,1)_{\mathrm{A}}$ at $4469 \mathrm{~Hz}$ and $(6,1)_{\mathrm{B}}$ at $4494 \mathrm{~Hz}$ are shown in Fig. 5. Also shown are their $1 / 3$ subharmonics at $1489 \mathrm{~Hz}$ and $1498 \mathrm{~Hz}$. The subharmonics have the same general appearance as their parents but, though the driving amplitudes were of equivalent level, the response was considerably lower. The existence of these, and other, subharmonics confirms the non-linear nature of the system.

\section{B. LDV and acoustic measurements}

To complement the ESPI measurements a Polytec Compact Laser Vibrometer, which measures velocity in the direction of the incident laser beam, was used to investigate the vibratory motion at a number of sensibly selected places on the surface of the gong. The laser beam was tightly focused onto the surface to a spot with circa $1 \mathrm{~mm}$ diameter

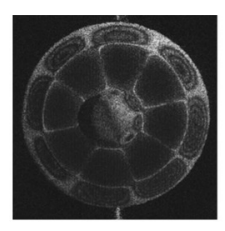

$(4,1)_{\mathrm{A}} 3044 \mathrm{~Hz}$

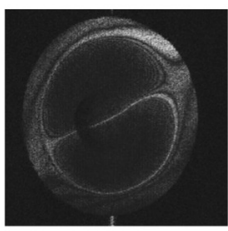

$(1,1)_{A} 697 \mathrm{~Hz}$

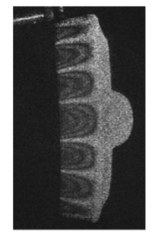

$(8,0) 3594 \mathrm{~Hz}$

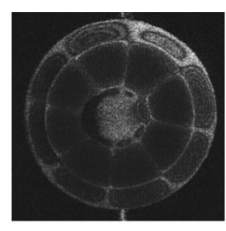

$(4,1)_{\mathrm{B}} 3089 \mathrm{~Hz}$

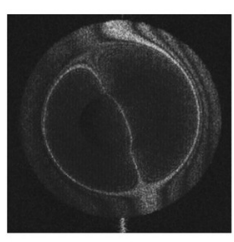

$(1,1)_{\text {B }} 734 \mathrm{~Hz}$

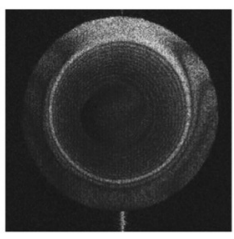

$(0,1) 458 \mathrm{~Hz}$
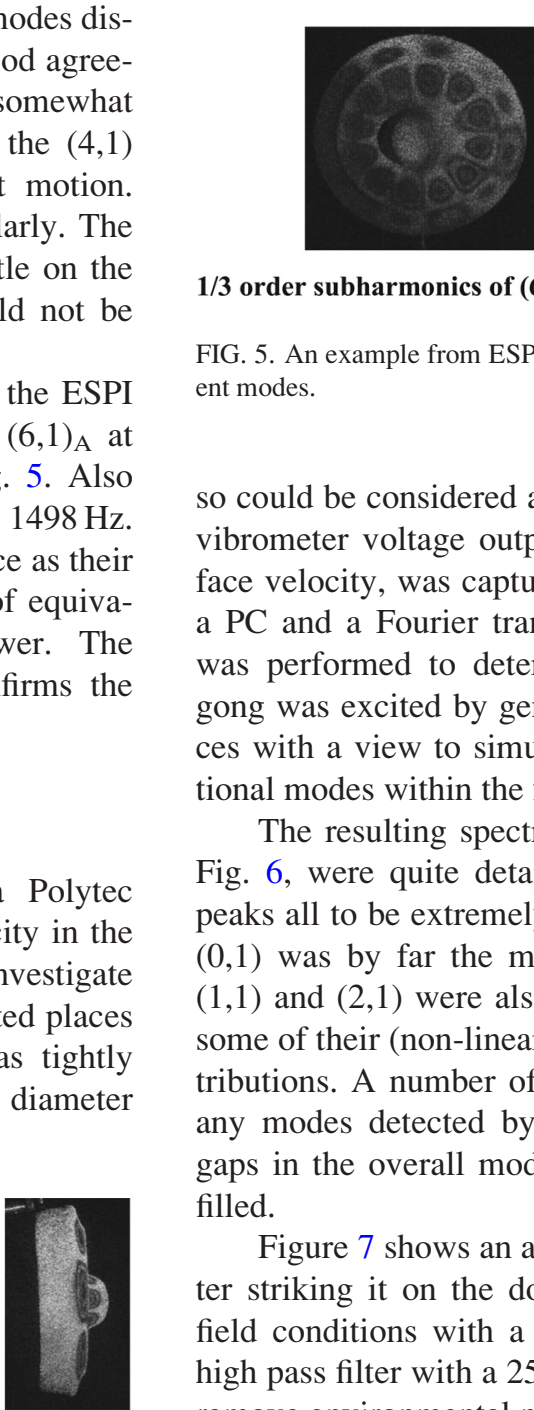

$(6,1)_{\text {B }} 4493.7 \mathrm{~Hz}$

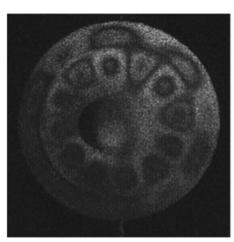

$(6,1)_{B}$ at $1489.7 \mathrm{~Hz}$ and $1498.0 \mathrm{~Hz}$

FIG. 5. An example from ESPI of a pair of $1 / 3$ subharmonics with their parent modes.

so could be considered as taking "point" measurements. The vibrometer voltage output, directly proportional to the surace velocity, was captured via a data acquisition system on PC and a Fourier transform, implemented using MATLAB, was performed to determine the frequencies present. The gong was excited by gentle impacts at carefully chosen places with a view to simultaneously exciting all of the vibrational modes within the frequency range of interest.

The resulting spectra, an example of which is given in Fig. 6, were quite detailed and showed the main resonant peaks all to be extremely sharp. Not surprisingly, the singlet $(0,1)$ was by far the most important mode. The two pairs $(1,1)$ and $(2,1)$ were also significant. Only $n=1$ modes and some of their (non-linear) harmonics made measureable conutions. A number of peaks found did not correspond to ESPI. These enabled some of the gaps in the overall mode data, as predicted by FEM, to be led. e. This was obtained under free6 pole zero-phase shift Butterworth 列 singlet $(0,1)$ was by far the most important mode. The split doublet pair $(1,1)$ were also significant. The figure clearly shows that any other frequencies involved decay away comparatively rapidly.

No evidence of a second important axisymmetric mode one octave above the $(0,1)$, as reported by Rossing using a small Balinese gong, was found. It is possible that he may have been observing a $(0,2)$ mode. However, in the present ESPI experiments, while a true $(0,1)$ mode was found at $458 \mathrm{~Hz}$ (with a [1/2] subharmonic having a closely similar but much fainter pattern at $230 \mathrm{~Hz})$, the $(0,2)$ mode was found at $1284 \mathrm{~Hz}$. This is very remote from the $(0,1)$ octave.

\section{Comparison of frequencies}

The frequency predictions of the FEM and the corresponding ESPI measurements are shown in Figs. 8 and 9 for $n=0$ and $n=1$, respectively. Frequencies above $5 \mathrm{kHz}$
FIG. 4. Selected modes observed using electronic speckle-pattern interferometry. 


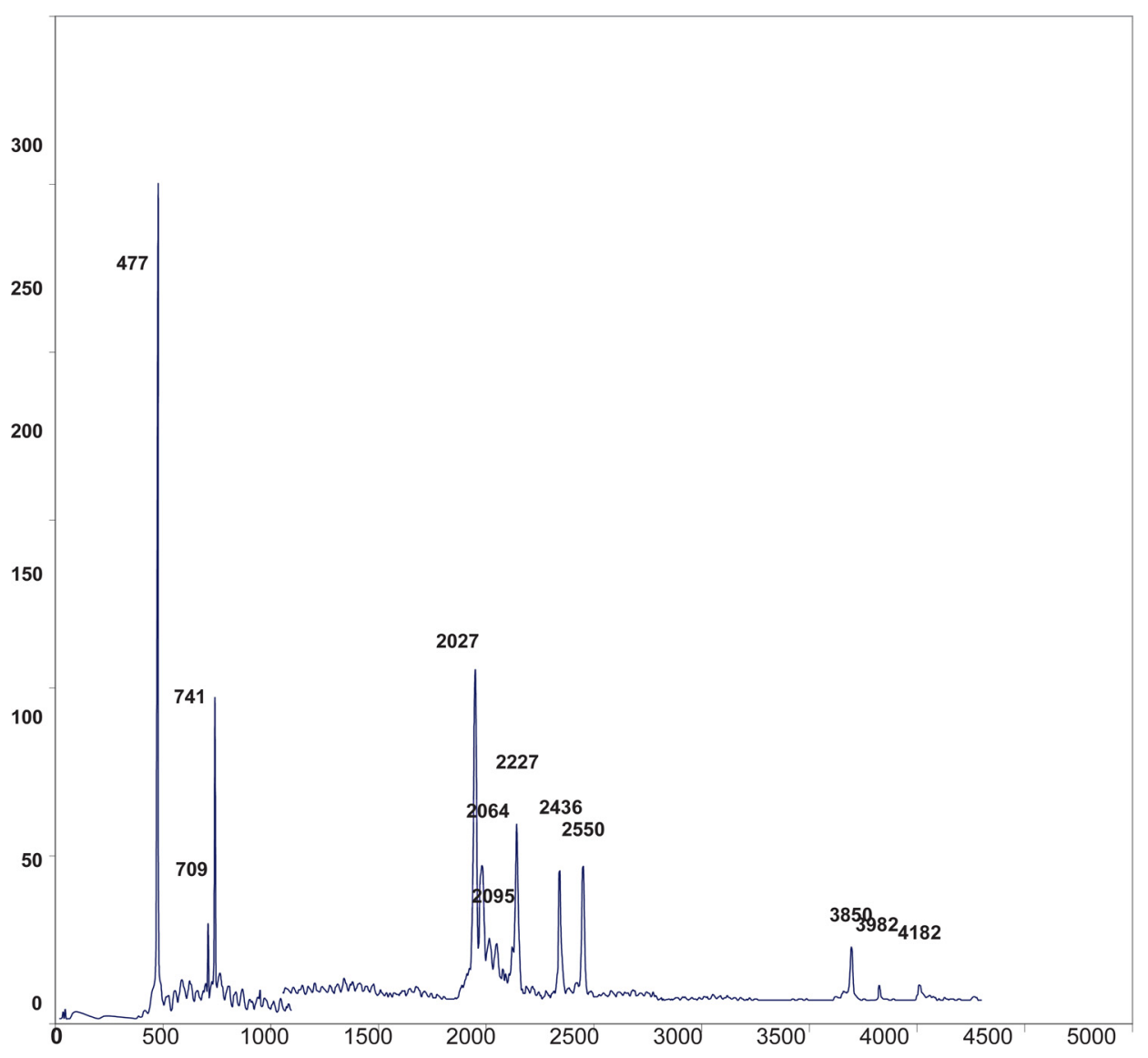

FIG. 6. (Color online) Example of an LDV spectrum for the gong struck and detected at a discrete point.

have been excluded because of the problems in interpreting their ESPI forms. Since the $n=0$ "rim" doublet modes were significantly split, only the higher frequency components are shown, for the sake of clarity. Clearly the agreement is excellent. In the case of the $n=1$ "plate" modes, the higher frequency components of the doublets are again the only ones shown. In this case the agreement was not nearly so good, although the trends were identical. To emphasize this, the FEM predictions, as plotted, have been reduced by $20 \%$ in the graph. Such a deviation could easily be caused by variations in the thickness of the top plate, due to hammering, making the model too crude an approximation. The $n=0$ modes depend hardly at all upon the details of the top plate, it being a region of evanescence for them. Rather they are expected to depend mainly on the details of the rim's geometry. Apart from the $(0,1)$ all the modes included in these figures are of the inextensional type as described in Sec. III B.

\section{Non-linearity}

Non-linear behavior is well established as being an important feature in many musical instruments. ${ }^{19}$ In the case of percussion instruments, it is usually associated with large amplitudes of vibration and has been reported in

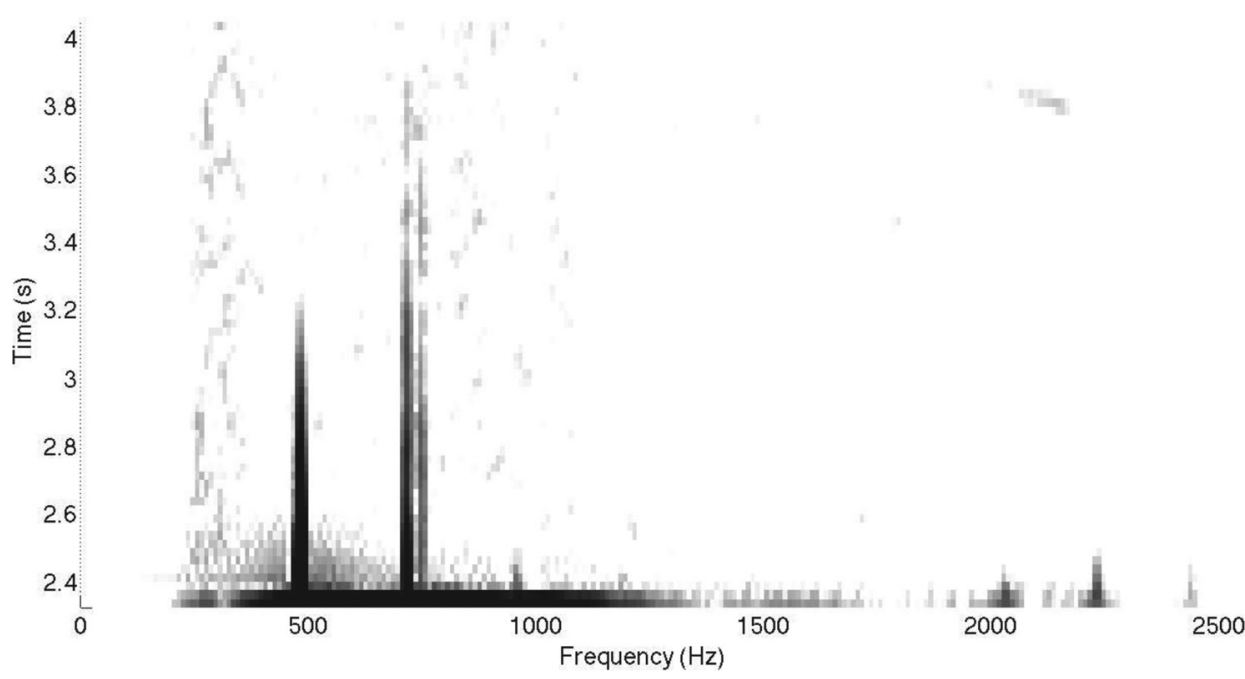

FIG. 7. Acoustic spectral map after striking on the dome showing the relative importance and decay of various natural frequencies. 


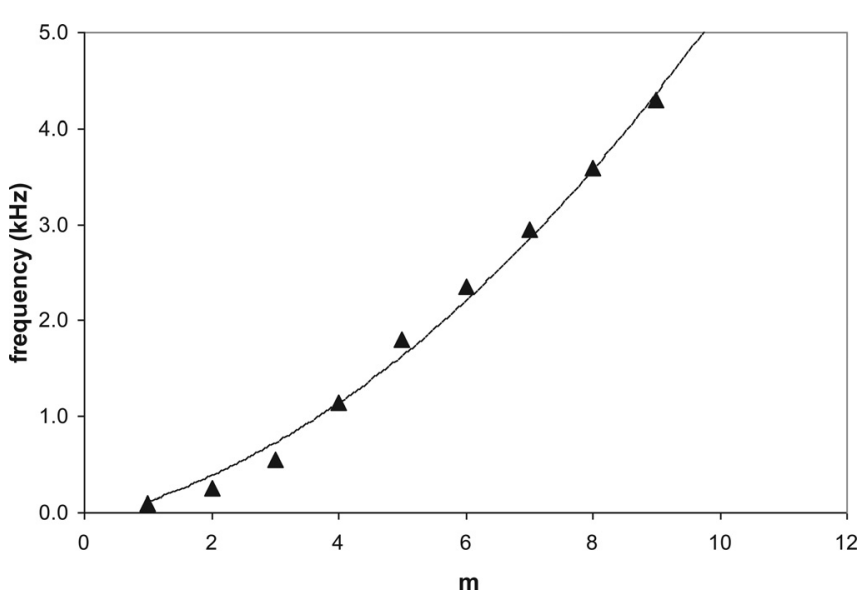

FIG. 8. Frequency vs $m$ for $n=0$ (higher frequency components) modes for ESPI ( $\mathbf{\Delta})$ and FEM (continuous line).

large-diameter examples of cymbals, shallow gongs, ${ }^{20}$ and gamelan gongs. ${ }^{3}$ Small gamelan gongs, being less flexible than their larger cousins, seem less likely to achieve sufficiently large amplitudes to produce non-linear effects. However, the ESPI studies revealed numerous "modes" over and above those expected from the FEM calculations. Nearly all of these proved to be subharmonics of parent modes. A typical example was discussed in Sec. V A. Only a few harmonics were found. No subharmonics appeared for any of the $(m, 0)$ modes apart from the $(1,0)$ pair which both showed $1 / 2$ examples. The $(m, 1)$ cases up to $m=7$ all showed clear subharmonics in pairs. For $m \geq 4$ they all showed $1 / 3$ subharmonics, for $m=0,1$ they showed $1 / 2$, while for $m=3$ both $1 / 2$ and $1 / 3$ were present The few $(m, 2)$ modes identified all had either a $1 / 2$ or a $1 / 3$ subharmonic.

The reason for the non-appearance of subharmonics for most of the $(m, 0)$ cases is not hard to see. For $m \geq 2$ they involve almost no motion on the top plate due to evanescence and so are restricted to the rim where large amplitudes are improbable. Only when $n \geq 1$ does the top plate participate significantly, making it possible for the amplitudes to become large enough to generate non-linear behavior.

Non-linear behavior was not observed acoustically. This was as expected with such a small gong being struck with a mallet. The energy input being spread across the whole spectrum; amplitudes of parent modes would simply be too low to generate non-linear behavior. With ESPI however,

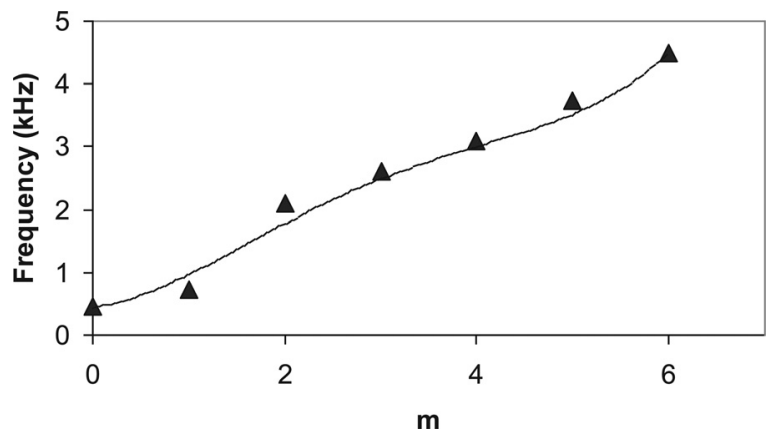

FIG. 9. Frequency vs $m$ for $n=1$ (higher frequency components) modes for $\operatorname{ESPI}(\mathbf{\Delta})$ and FEM (continuous line). because a monochromatic signal with very high amplitude was used, such behavior was readily observed.

\section{E. Chladni's Law}

In the study of the normal modes of flat circular plates, a law first proposed by Chladni ${ }^{21}$ was given some mathematical justification by Lord Rayleigh. ${ }^{22}$ His formulation showed that, under asymptotic conditions, the frequency $f_{m, n}$ of a normal mode with $m$ nodal diameters and $n$ nodal circles should be given by

$$
f_{m, n} \approx C(m+2 n)^{2}
$$

where $C$ is a known constant. It was shown by Rossing, ${ }^{23}$ on analyzing the extensive data of Waller, ${ }^{24}$ that while this equation does not work particularly well, it can be improved by modifying it into

$$
f_{m, n}=C_{n}(m+2 n)^{P n},
$$

where $C_{n}$ and $P_{n}$ are constants for a given plate with $P_{n}$ not differing much from 2. This equation, sometimes called "modified Chladni's law," has been applied with varying success to other axially symmetric systems including thin rings 9 and church bells. ${ }^{25}$ It has to be remembered that, because of the asymptotic nature of the underlying derivation, the equation cannot be expected to work for low values of $(m+2 n)$ which means, in the present context, low values of $m$.

Because of its limited success with other axisymmetric systems it is of interest to try to apply it to the present small gamelan gong. Sufficient data was available only for the $n=0$ and $n=1$ cases. As a trial $P_{n}$ was set to 2 for both $n$ values. The resulting fits are shown in Fig. 10 with the lower values of $m$ omitted. In each case only the higher components of the doublet pairs have been used. The fits were extremely good and the resulting parameters are included in Table I. From the table it can be seen that the fits to the lower frequency components are even better than those in the figure. The fact that the fits do not pass through the origin is a reflection of the asymptotic nature of the "law" and the remoteness of the gong from a flat circular plate. One could

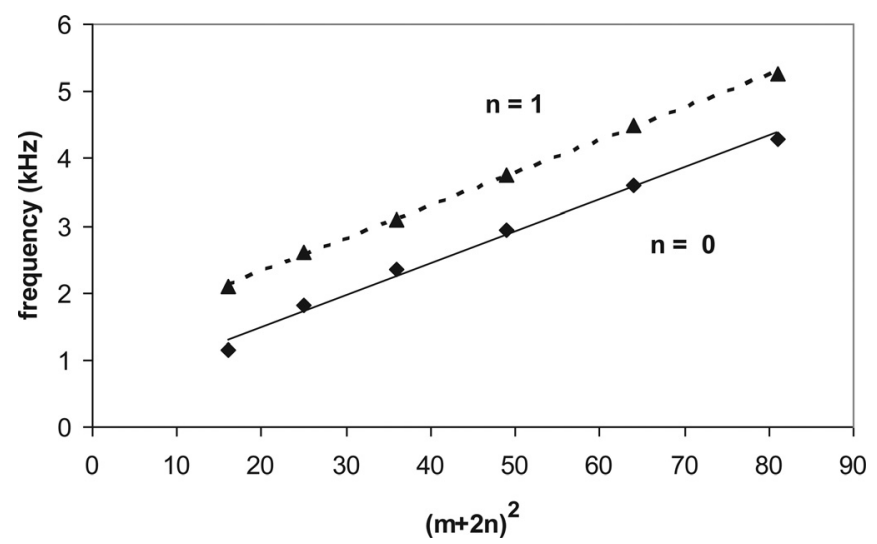

FIG. 10. Frequency vs $(m+2 n)^{2}$ for $n=0,1$ (upper components) with low $m$ excluded. 
TABLE I. Chladni's law fits to the ESPI data.

\begin{tabular}{lcccc}
\hline \hline Mode family & Components & $m$ range fitted & $C_{n}$ & $R^{2}$ \\
\hline$n=0$ & upper & $4-9$ & 47.26 & 0.9925 \\
& lower & $4-9$ & 49.87 & 0.9894 \\
\multirow{2}{*}{$n=1$} & upper & $2-7$ & 48.70 & 0.9994 \\
& lower & $2-7$ & 48.23 & 0.9966 \\
\hline \hline
\end{tabular}

bring the two linear fits in Fig. 10 into coincidence by adjusting the factor 2 on the right hand side of Eq. (4) but it is hard to see what the physical significance of this might be.

\section{SCANNING LDV MEASUREMENTS}

In order to check the ESPI results further, it was decided to conduct a new set of experiments with the same gong, supported similarly, but now using scanning LDV. This technique, conceived some decades ago, ${ }^{26}$ is, like ESPI, used in a number of sectors as an alternative to the more traditional contact vibration measurement methods but has specific advantages for measurements such as those conducted in this study. ${ }^{27}$ It involves scanning a laser beam across the surface of the structure of interest, addressing points of interest sequentially, using a pair of orthogonally aligned mirrors. Directing the beam onto the top surface of the gong parallel to the symmetry axis enabled the motion in that direction to be studied. Directing the beam normally to the axis onto the rim, by using an angled mirror, enabled the motion there to be investigated during the same measurement.

\section{A. Experimental details}

The facility used to perform these experiments has been described in some detail previously. ${ }^{4}$ The gong was suspended in this case by a light elastic band around its widest point and the driving point chosen such that there was expected to be activity in most of the modes of interest. A miniature force transducer was attached to the gong surface to measure the (reference) input signal. Excitation was generated using a permanent magnet electrodynamic shaker connected to the force transducer through a pin vice and thin wire "stinger" arrangement. A Polytec (2D) scanning LDV system was used to measure the vibratory response of the gong at a discrete series of points on the surface. Broadband (white) noise, generated within the scanning LDV system with an appropriate frequency range, was amplified using an LDS PA25E power amplifier and used to drive the shaker. Bursts of random excitation were used with a $5 \%$ block length build-up and a 50\% of block-length burst. These parameters gave a gong response that decayed within the acquisition block such that a rectangular acquisition window could be used without significant risk of spectral leakage. Twenty linear averages were used to maximize the signal-tonoise ratio in the measured mobility frequency response functions (FRFs). Typical experimental modal analysis good practice $^{28}$ was employed to ensure that high quality data could be realized.

Response measurement points were directly defined in the scanning LDV software on a regular grid with enough spatial resolution to enable representation of the mode shapes at all frequencies of interest. The scanning LDV system was positioned $5 \mathrm{~m}$ from the gong in order that the angle of incidence of the laser beam was never more than $5^{\circ}$ off axis such that the sensitivity to velocity normal to the intended measurement direction was negligible. The frequency range of interest and required specified settings often led to acquisition periods of several hours. Care was taken to ensure that the ambient conditions and other environmental effects did not impact significantly on the quality of the measured data.

Modal processing of the FRFs was performed in the Polytec scanning vibrometer software by manually defining multiple search frequency bands within the sum FRF, from which the peak amplitudes were automatically identified. A least squares complex exponential curve fitting algorithm was subsequently used to synthesize a number of single degree of freedom damped exponentials from which the Eigenfrequencies were extracted.

\section{B. Scanning LDV results and discussion}

The identification of the gong's modes using scanning LDV was relatively easy compared with ESPI. Fewer modes were detected, but this was partly because relatively few harmonics and subharmonics were excited. As expected, modes became increasingly difficult to find and identify as the frequency increased. Again some modes were badly distorted and there was some evidence of mode-mixing. Similarly to ESPI, most $n=0$ modes could only be detected by looking at the side-on view. In Fig. 11 a selection of the modal forms

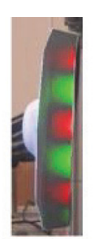

$3580 \mathrm{~Hz}(5,1)$

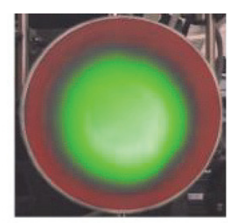

$470 \mathrm{~Hz}(0,1)$

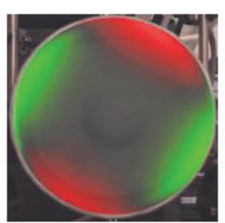

$516 \mathrm{~Hz}(2,0)$

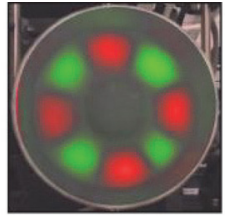

$3008 \mathrm{~Hz}(4,1)_{\mathrm{A}}$

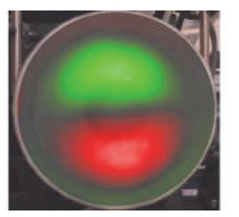

$723 \mathrm{~Hz}(1,1)_{\mathrm{A}}$

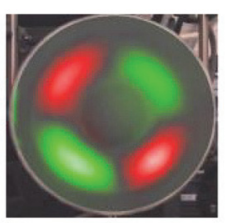

$1986 \mathrm{~Hz}(2,1)$

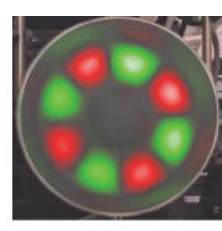

$3073 \mathrm{~Hz}(4,1)_{\text {В }}$

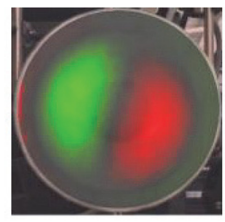

$745 \mathrm{~Hz}(1,1)_{B}$

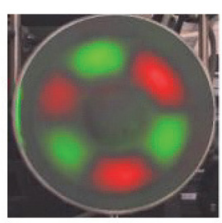

$2556 \mathrm{~Hz}(3,1)$
FIG. 11. (Color online) Selected modes observed using scanning laser Doppler vibrometry. 


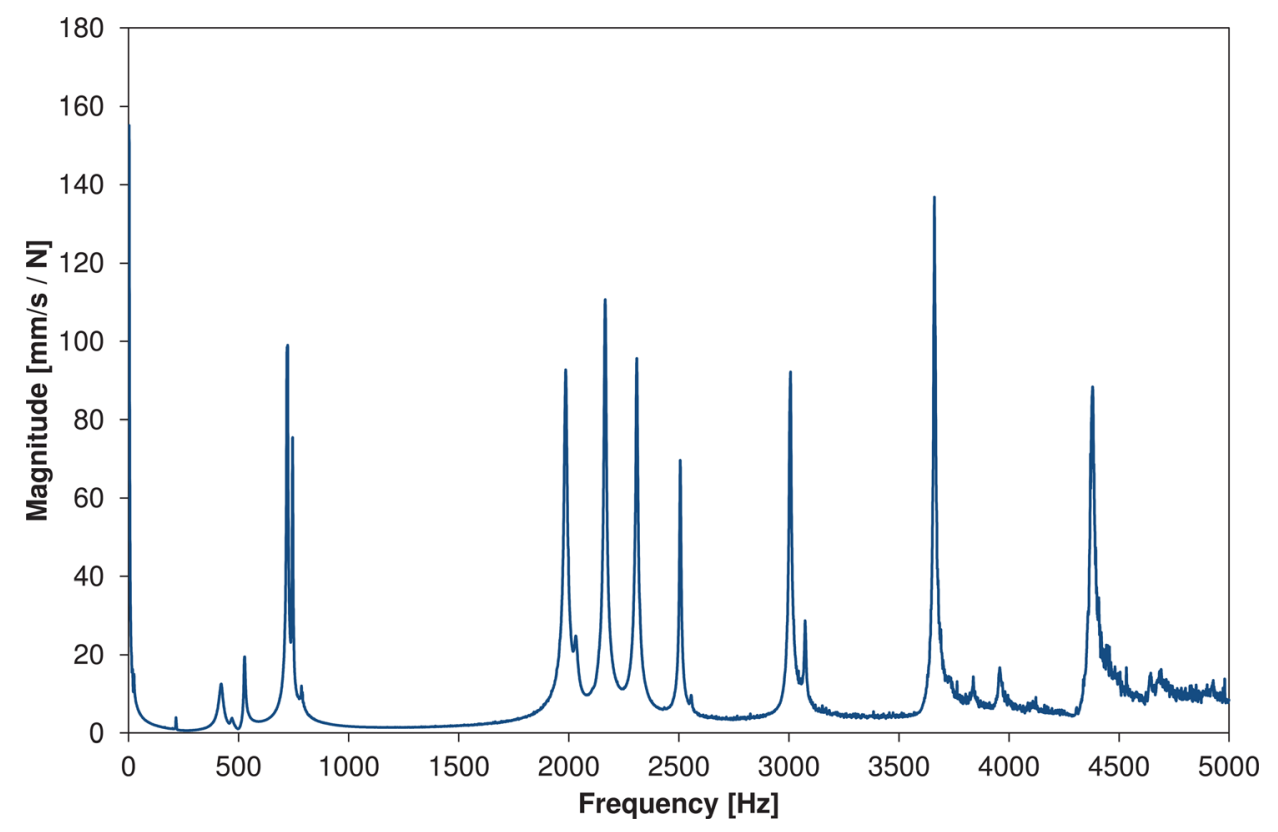

FIG. 12. (Color online) Example scanning LDV frequency response function for the gong.

from scanning LDV are shown. When the top row is compared with those of Figs. 3 and 4 the agreement for the $(4,1)$ modes is seen to be striking, except that scanning LDV shows relatively little motion on the shoulder. This could be attributed to the fact that, as previously described, scanning LDV measures velocity in the direction of the laser beam. Given the geometry of the gong, the shoulder's motion may be much more significant in directions not closely aligned with the incident beam direction. The forms shown in the second row of Fig. 11 are in even better agreement with those in Figs. 3 and 4. In these cases there is little shoulder motion, as expected. In the third row members of the $(2,0)$ and $(2,1)$ pairs are included in order to emphasize the differences between them.

An example scanning LDV spectrum for the gong is shown in Fig. 12. Comparing this with Fig. 6 one sees good overall agreement between the modal frequencies although, as expected, the relative magnitudes of the peaks differ. Also the scanning LDV spectrum shows up the $(4,1)$ modes which do not appear in the LDV spectrum, although they were easily detected by ESPI. Table II shows a comparison of the frequencies measured from ESPI and scanning LDV.

\section{COMPARISON OF METHODS}

For both ESPI and scanning LDV methods the detection of modes, being optical, is non-contact. In ESPI the gong was excited acoustically with a sinusoidal signal whose frequency was varied manually and tuned to find amplitude peaks. This introduced uncertainties into the measured frequencies as the peaks were sometimes rather broad. It also made the experiments long and tedious. With scanning LDV, on the other hand, the gong was excited mechanically with "white noise" at a single point and the frequencies were extracted by the software. This could also take a long time but had the advantage of being essentially automated. ESPI was more sensitive as a detector of modes and found numerous subharmonics of true modes, while scanning LDV found relatively few.

Provided the mode order did not become too high it was, in general, not difficult to interpret the ESPI interferograms on the top plate and on the shoulder. When looking towards the rim it was sometimes difficult to identify $m$ values but it could usually be done. With scanning LDV it was easy to see what was happening on the top plate but with the shoulder it was problematic. When looking at the rim using scanning LDV it was not too difficult to establish the $m$ values, but relatively few $n=0$ cases could be found. This could be attributed to a number of things including (1) a suboptimal choice of driving point and (2) the fact that bursts of random excitation were used to excite all modes simultaneously with a significantly lower input of energy per mode than in the case of ESPI. This reduced energy per mode may also account for the relatively few cases of subharmonics and other non-linear effects.

TABLE II. Comparison of measured frequencies.

\begin{tabular}{lcc}
\hline \hline Modes & ESPI $(\mathrm{Hz})$ & Scanning LDV (Hz) \\
\hline$(0,1)$ & 458 & 470 \\
$(1,1)$ & 698,734 & 723,745 \\
$(2,1)$ & 2005,2093 & 1986,2031 \\
$(3,1)$ & 2529,2606 & 2506,2556 \\
$(4,1)$ & 3045,3089 & 3008,3073 \\
$(5,1)$ & 3726,3748 & 3580,3661 \\
$(6,1)$ & 4469,4494 &,-- \\
$(1,0)$ & 69,99 &,-- \\
$(2,0)$ & 240,244 & $216,-$ \\
$(3,0)$ & 527,556 & 528,545 \\
$(4,0)$ & 945,1151 & $975,-$ \\
$(5,0)$ & 1388,1807 & 1441,1788 \\
$(6,0)$ & 2227,2344 & 2299,2324 \\
$(7,0)$ & $2941,-$ & $2920,-$ \\
$(8,0)$ & 3436,3594 &,-- \\
$(9,0)$ & 4182,4300 &,-- \\
\hline \hline
\end{tabular}


Some of the discrepancies between the frequencies measured by the two methods, as shown in Table II, are larger than one might wish. There are at least two possible reasons for this. First the ESPI method induced considerable non-linear behavior, which could have caused some frequency shifts away from the "true" linear values. Second, the use of a mechanical driver in scanning LDV will have caused slight changes in the mass and stiffness of the system which could also result in frequency changes. Overall the agreement between ESPI and scanning LDV is considered reasonable although scanning LDV detected fewer modes. Given that the two methods use very different approaches, it is encouraging that the results are in such reasonable agreement concerning "true" modes.

\section{CONCLUSIONS}

The normal modes of a $20.7 \mathrm{~cm}$ diameter steel gamelan gong are now reasonably well understood and the acoustically important ones identified. Expectations from slightly broken axial symmetry were well satisfied and similarities with cymbals, bells, and other axisymmetric systems are clear. Non-linear behavior, mainly in the form of subharmonics of true modes, has been established. The experimental frequencies have been shown to satisfy a modified version of Chladni's law and also to agree tolerably well with the predictions of a finite-element model. A comparison of ESPI and scanning LDV methods has established that the latter is also a useful technique for investigating these gongs, especially in identifying the acoustically important low order modes.

${ }^{1}$ N. H. Fletcher and T. D. Rossing, The Physics of Musical Instruments, 2nd ed. (Springer-Verlag, New York, 1998), p. 645.

${ }^{2}$ T. D. Rossing, Science of Percussion Instruments (World Scientific, Singapore, 2000), pp. 98-99.

${ }^{3}$ D. W. Kruger, K. L. Gee, and J. Grimshaw, "Acoustical and vibrometry analysis of a large Balinese gamelan gong," J. Acoust. Soc. Am. 128, EL8-EL13 (2010).

${ }^{4}$ R. Perrin, S. Hamden, B. J. Halkon, and G. M. Swallowe, "Studies with a small gamelan gong," Proc. Inst. Acoust. 35(1), 337-344 (2013).

${ }^{5}$ N. McLachlan, "Finite element analysis and gong acoustics", Acoust. Aust. 25(3), 103-107 (1997).
${ }^{6}$ R. Perrin and T. Charnley, "Group theory and the bell," J. Sound Vib. 31, 411-418 (1973).

${ }^{7}$ R. Perrin, G. M. Swallowe, T. R. Moore, and S. A. Zietlow, "Normal modes of an 18 inch crash cymbal," Proc. Inst. Acoust. 28, 653-662 (2006).

${ }^{8}$ A. W. Leissa, Vibration of Plates (NASA, Washington, DC, 1973) [reprinted (Acoustical Society of America, Woodbury, NY, 1993), Chap. 2, pp. 7-37].

${ }^{9}$ T. D. Rossing and R. Perrin, "Vibrations of bells," Appl. Acoust. 20, 41-70 (1987).

${ }^{10}$ T. Charnley, R. Perrin, V. Mohanan, and H. Banu, "Vibrations of thin rings of rectangular cross-section," J. Sound Vib. 134, 455-488 (1989).

${ }^{11}$ J. W. Strutt (Lord Rayleigh), Theory of Sound, 2nd ed. (Dover, New York, 1945), Vol. 1, Chap. $X_{\text {A. }}$

${ }^{12}$ R. Perrin, T. Charnley, and J. dePont, "Normal modes of the modern English church bell," J. Sound Vib. 90, 29-49 (1983).

${ }^{13}$ R. Perrin, L. Chalmers, D. P. Elford, G. M. Swallowe, and T. R. Moore, "Normal modes of the Indian elephant bell," J. Acoust. Soc. Am. 131(3), 2288-2294 (2012).

${ }^{14}$ R. Perrin and H. P. W. Gottlieb, "Evanescence and Bessel functions in the vibrating circular membrane,” Eur. J. Phys. 15, 293-299 (1994).

${ }^{15}$ R. Jones and C. Wykes, Holographic and Speckle Interferometry, 2nd ed. (Cambridge University Press, New York, 1989), pp. 165-196.

${ }^{16}$ W. Kausel, D. W. Zeitlow, and T. R. Moore, "Influence of wall vibrations on the sound of brass wind instruments," J. Acoust. Soc. Am. 128, 3161-3174 (2010).

${ }^{17}$ B. M. Deutsch, C. I. Ramirez, and T. R. Moore, "The dynamics and tuning of orchestral croatals," J. Acoust. Soc. Am. 116, 2427-3433 (2004).

${ }^{18}$ T. R. Moore and S. A. Zeitlow, "Interferometric studies of a piano soundboard," J. Acoust. Soc. Am. 119, 1783-1793 (2006).

${ }^{19}$ N. H. Fletcher, "The non-linear physics of musical instruments," Rep. Prog. Phys. 62, 723-764 (1999).

${ }^{20}$ K. A. Legge and N. H. Fletcher, "Non-linearity, chaos and sound of shallow gongs," J. Acoust. Soc. Am. 86(6), 2439-2443 (1989).

${ }^{21}$ E. F. F. Chladni, Entdeckungen über die Theorie des Klanges (Discoveries in the Theory of Sound) (Breitkopf and Härtel, Leipzig, 1787), pp. 1-77.

${ }^{22}$ J. W. Strutt (Lord Rayleigh), Theory of Sound (Ref. 11), Vol. 1, Sec. 218.

${ }^{23}$ T. D. Rossing, "Chladni's law for vibrating plates," Am. J. Phys. 50, 271-274 (1982).

${ }^{24}$ M. D. Waller, "Vibrations of free circular plates," Proc. Phys. Soc. London 50, 70-86 (1938).

${ }^{25}$ R. Perrin, T. Charnley, H. Banu, and T. D. Rossing, "Chladni's law and the modern English church bell," J. Sound Vib. 102, 11-19 (1985).

${ }^{26}$ P. Sriram, J. I. Craig, and S. Hanagud, "A scanning laser Doppler Vibrometer for modal testing," Int. J. Anal. Exp. Modal Anal. 5(7), 155-167 (1990).

${ }^{27}$ S. J. Rothberg, B. J. Halkon, M. Tirabassi, and C. Pusey, "Radial vibration measurements directly from rotors using laser vibrometry: the effects of surface roughness, instrumental misalignment, and pseudo-vibration," Mech. Syst. Sign. Process. 33(11), 109-131 (2012).

${ }^{28}$ D. J. Ewins, Modal Testing: Theory, Practice and Applications, 2nd ed. (Research Studies Press, Baldock, England, 2000), pp. 163-276. 